\title{
Science in the open
}

\author{
As moves towards open-access schemes gain momentum, the choice between 'author pays' and \\ subscription-based models may come down to fundamental business considerations rather than limits \\ in access to original research.
}

The scientific discourse is, by its very nature, open and democratic. Anyone can participate, and, based on the principles of deductive logic and experimental discovery, anyone can contribute to the process. There is no place for censorship in science. As an essay in the New York Times makes clear, "If we are not practicing good science, we probably aren't practicing good democracy. And vice versa" ${ }^{\text {. }}$.

The natural consequence of this openness in the practice of science is that all aspects of science should be open and accessible to everyone. In that spirit, several universities, including Harvard ${ }^{2}, \mathrm{MIT}^{3}$ and University College London ${ }^{4}$, have recently announced initiatives that aim to make the scientific output of their researchers freely available in public repositories.

These schemes are part of a more general drive towards open access in publishing, whereby authors, not readers, pay for publication costs, and access and dissemination of those papers is free. At Nature Publishing Group, we recognize these trends. Our authors of original research papers retain copyright to their work $^{5}$, with important implications. For example, at Nature Materials, as well as other Nature journals, we allow authors to post presubmitted or submitted versions of a paper on preprint servers such as arXiv or Nature Precedings. We also allow these authors to reuse papers in their future printed work, and research papers can be archived in public repositories such as PubMed Central six months after publication. In addition, some of our academic journals, such as Molecular Systems Biology, are fully open access.

So why not implement full open access for all of our journals? Practical considerations have an important role. According to Journal Citation Reports (Thomson Reuters), in 2007 we published 133 research papers (including review articles). Subscription-based Advanced Materials published 736 items, and the open-access journal Optics Express published 1961 papers, with publication fees starting from $\$ 925$ per paper. From such statistics it is obvious that, assuming simple economics of scale, a switch to an author-pays model in our case would incur significant publication fees per paper published.

In addition, at every stage of manuscript handling we provide an expensive, highquality service. This not only involves the professional subediting and production of accepted papers, but also an exhaustive prescreening of submitted manuscripts. At Nature Materials, we prescreen well above $80 \%$ of submitted manuscripts without peer review. This means that, at a cost, we rely much less on the 'free' peer-reviewing services of scientists than journals with lower screening rates.

As open access certainly should not be considered as a way to lower publication standards, the overall expenses related to the dissemination of scientific results should be considered so that the costs remain the same. This means that researchintensive institutions in particular (or those paying for their research grants) may well end up paying proportionally more under author-pays models than they would under subscription-based models. Researchers from less researchintensive institutions on the other hand would benefit.

Of course, even in a subscription-based model, science needs to be open, and researchers from less-developed countries in particular should not be disadvantaged by being asked to pay beyond their means for journal subscriptions. For that reason, Nature Publishing Group is in partnership with a number of initiatives that provide free access to journal content to scientists from such countries ${ }^{6}$.

Efforts like these, in addition to the freedom that we grant to our authors in making their research available in public repositories after a reasonable amount of time, illustrate that the choice between open access and a subscription-based approach is more a matter of having a sustainable business model, rather than that of a fundamentally different point of view. Sharing our entire publication costs among only 133 authors, rather than thousands of subscribing individuals and institutions, certainly does not seem feasible.

As for the openaccess resolutions by universities such as Harvard and MIT, at present we do not wish to accept any addenda to our author licence, because, as outlined above, we already provide comprehensive rights to our authors. Instead, before publication we ask authors from those institutions to provide us with an exemption to their policy, as foreseen in these open-access resolutions.

At Nature Publishing Group we will continue to deliver free services to the scientific community, ranging from our social networking website Nature Network, to reference-management tools such as Connotea, as well as weekly podcasts. To further strengthen our engagement with our own research community, Nature Materials editorials are now freely available to any registered user of our website. This follows a similar decision taken at Nature some years ago. The free access also applies to our back catalogue of editorials, and we hope you enjoy reading them, whether through a subscription or through free online access!

\footnotetext{
References

1. www.nytimes.com/2009/01/27/science/27essa.html

2. http://cyber.law.harvard.edu/node/3462

3. http://web.mit.edu/newsoffice/2009/open-access-0320.html

4. www.ucl.ac.uk/media/library/OpenAccess

5. www.nature.com/authors/editorial_policies/license.html

www.nature.com/info/partners.html
} 